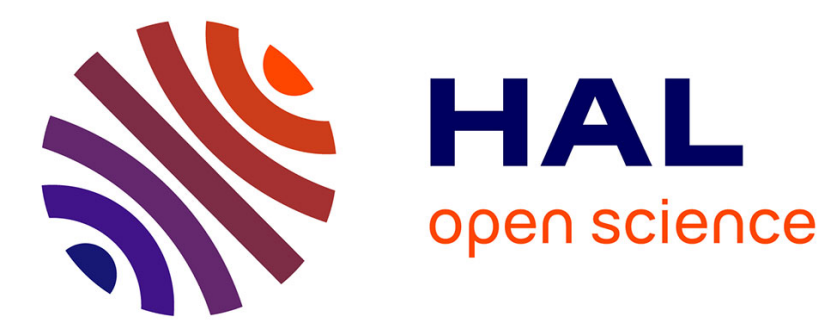

\title{
L'avenir incertain des marques de distributeurs
}

Odile Chanut, Gilles Paché

\section{To cite this version:}

Odile Chanut, Gilles Paché. L'avenir incertain des marques de distributeurs. Expansion Management Review, 2010, 138 (3), 10.3917/emr.138.0010 . hal-01767001

\section{HAL Id: hal-01767001 \\ https://hal.science/hal-01767001}

Submitted on 14 Apr 2018

HAL is a multi-disciplinary open access archive for the deposit and dissemination of scientific research documents, whether they are published or not. The documents may come from teaching and research institutions in France or abroad, or from public or private research centers.
L'archive ouverte pluridisciplinaire HAL, est destinée au dépôt et à la diffusion de documents scientifiques de niveau recherche, publiés ou non, émanant des établissements d'enseignement et de recherche français ou étrangers, des laboratoires publics ou privés. 


\section{L'AVENIR INCERTAIN DES MARQUES DE DISTRIBUTEURS Odile Chanut, Gilles Paché}

L'Express - Roularta | «'Expansion Management Review »

$2010 / 3 N^{\circ} 138 \mid$ pages 10 à 21

ISSN 1254-3179

Article disponible en ligne à l'adresse :

https://www.cairn.info/revue-l-expansion-management-review-2010-3-page-10.htm

\section{Pour citer cet article :}

Odile Chanut, Gilles Paché« L'avenir incertain des marques de distributeurs », L'Expansion Management Review 2010/3 ( $\mathrm{N}^{\circ} 138$ ), p. 10-21.

DOI 10.3917/emr.138.0010

Distribution électronique Cairn.info pour L'Express - Roularta.

(C) L'Express - Roularta. Tous droits réservés pour tous pays.

La reproduction ou représentation de cet article, notamment par photocopie, n'est autorisée que dans les limites des conditions générales d'utilisation du site ou, le cas échéant, des conditions générales de la licence souscrite par votre établissement. Toute autre reproduction ou représentation, en tout ou partie, sous quelque forme et de quelque manière que ce soit, est interdite sauf accord préalable et écrit de l'éditeur, en dehors des cas prévus par la législation en vigueur en France. Il est précisé que son stockage dans une base de données est également interdit. 


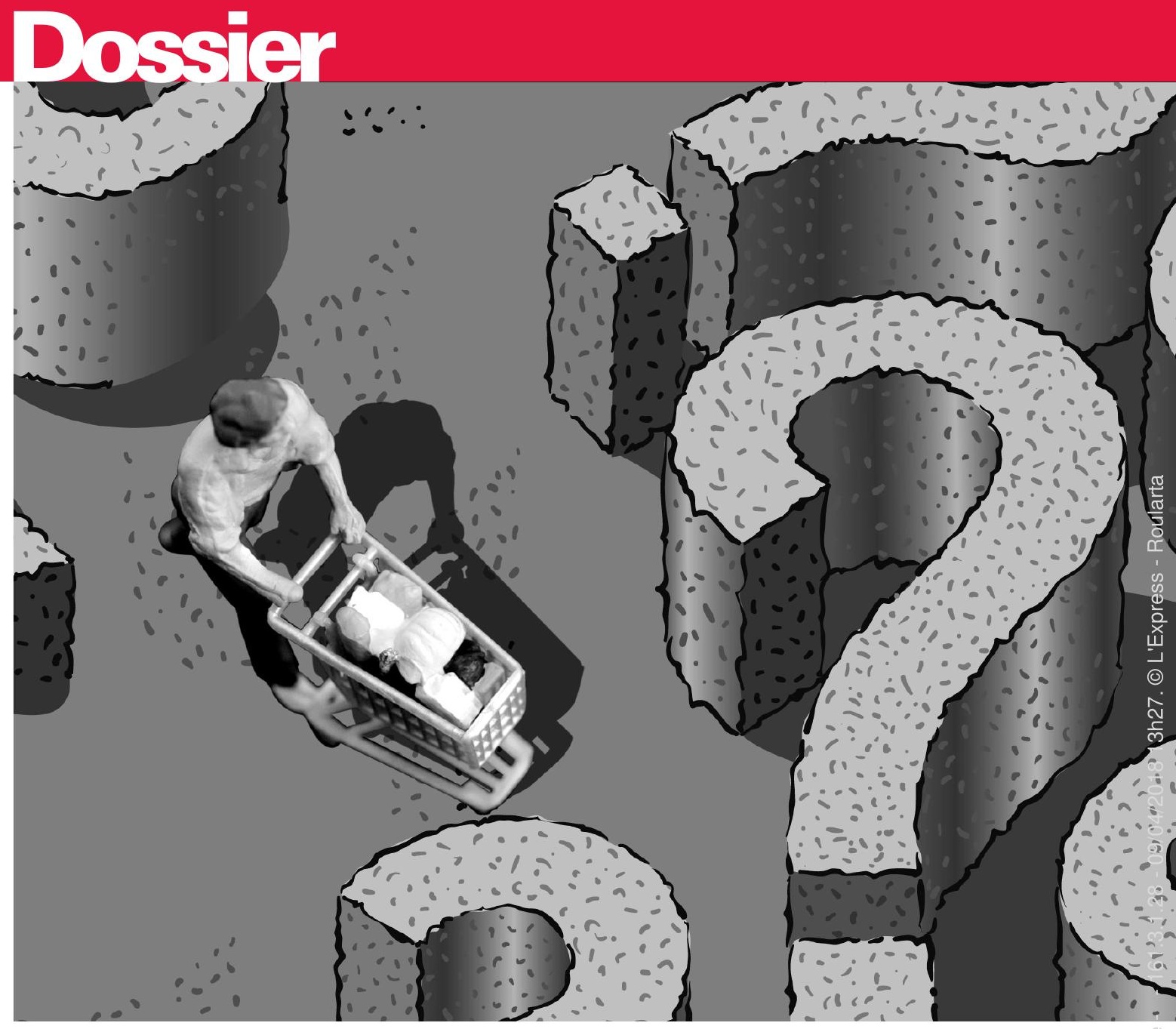

\section{L'avenir incertain des marques de distributeurs}

LES MARQUES DE DISTRIBUTEURS ONT QUITTÉ, PEU À PEU, LEURS HABITS DE COPIES DES GRANDES MARQUES POUR SE POSITIONNER SUR LE HAUT DE GAMME. MAIS LA CRISE EST LÀ...

\section{$>$ Odile Chanut et Gilles Paché}

a marque de distributeur (ou encore MDD) est souvent présentée comme une manière de vendre beaucoup moins cher des produits d'une qualité proche de celle des fabricants de marques nationales. La réalité des choix stratégiques des distributeurs semble pourtant de plus en plus éloignée de cet idéal consumériste.
En effet, si les " produits libres » lancés en avril 1976 par Carrefour visaient à faire aussi bien que les marques nationales mais avec un prix plus attractif, quitte à purement et simplement les copier (selon la logique $\mathrm{du}$ « me too product»), les évolutions ultérieures ont conduit à l'émergence de MDD thématiques positionnées haut de gamme et participant à la consolidation de l'image

Odile Chanut et Gilles Paché sont respectivement maître de conférences et professeur en sciences de gestion à l'université de la Méditerranée (Aix-Marseille 2). Ils appartiennent tous deux au Centre de recherche sur le transport et la logistique (CRET-LOG), localisé à Aix-en-Provence. 


\section{\$) Actualité du modèle de la roue de la dis-} tribution. Que recouvre ce modèle, formulé par Malcolm McNair (1) il y a plus de cinquante ans de cela? En s'inspirant d'une approche fondée sur la notion de cycle de vie, son objectif est de fournir un schéma original de l'évolution dans le temps des formats (le grand magasin, le supermarché, l'hypermarché, etc.). Lorsqu'un nouveau format commence à s'implanter, note-t-il, le succès attire des concurrents qui imitent les pratiques des innovateurs et leur disputent les clients. Les entreprises qui utilisent ce format cherchent alors à se différencier les unes des autres. Elles ne peuvent y parvenir qu'en élargissant leur assortiment et en proposant des services plus sophistiqués, ce que Malcolm McNair qualifie d'embourgeoisement. Il en résulte une augmentation des coûts de distribution, d'abord masquée par les économies d'échelle provoquées par le succès du format et l'augmentation des ventes qui en résulte. Quand l'ensemble de la clientèle susceptible d'être attirée par le format finit par être conquis, les effets bénéfiques de la croissance disparaissent et les coûts de différenciation obligent les entreprises à relever leurs prix pour préserver leur rentabilité. Parvenu à ce stade de développement, le format devient vulnérable et suc-

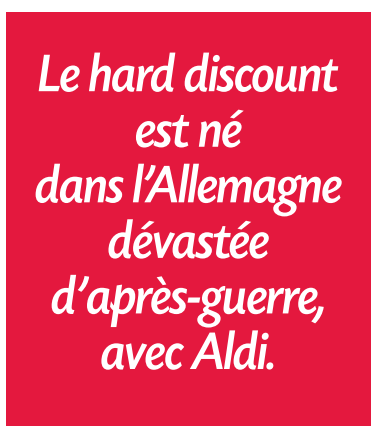

atteindre la fin des années 70 pour que le hard discount apparaisse en France, avec l'ouverture des magasins ED du groupe Carrefour. La vraie naissance du hard discount en France date cependant de l'arrivée d'Aldi en 1988, suivie de l'implantation de Lidl et Norma en 1989. Très rapidement, quelques grands distributeurs français qui gèrent des hypermarchés et des supermarchés réagissent en ouvrant leurs propres magasins de hard discount (CDM puis Netto pour Intermarché, Dia pour Promodès), mais la croissance du format de vente est essentiellement tirée, aujourd'hui encore, par les hard discounteurs allemands.

Le développement du format en France, confirmé depuis le ralentissement économique de l'année 2009 (qui a conduit le groupe Auchan à ouvrir son premier hypermarché hard discount en janvier 2010), pousse les différentes enseignes à rechercher de nouvelles sources de différenciation. Celles-ci consistent tout d'abord à élargir et enrichir progressivement l'assortiment, entre autres vers les produits frais. C'est notamment le cas de Franprix, qui a créé dans plusieurs magasins un coin « minute lunch » proposant des plats cuisinés. L'enseigne a également renforcé la place des produits frais. Alors que le modèle d'origine du hard discount se fondait sur une proposition commerciale tournant autour de 600 références, ce nombre ne va cesser combe parfois à l'attaque des concurrents.

La rapide croissance du format hard discount en France (et plus généralement en Europe), et les difficultés rencontrées par l'hypermarché, constituent une excellente illustration de la roue de la distribution. Le hard discount est né dans l'Allemagne dévastée de l'après-guerre, à l'initiative des frères Albrecht, créateurs d'Aldi. Leur volonté était de proposer aux consommateurs des produits alimentaires dans des magasins de petite taille ayant une présentation austère et peu soignée. Il faudra d'augmenter pour dépasser les 2000 références pour certaines enseignes. Mieux encore, les marques nationales, considérées comme persona non grata chez les hard discounteurs, finissent par occuper une place certes mineure, mais non négligeable (voir tableau ci-contre).

De nouveaux services au client sont introduits en magasin, tout particulièrement

> (1) M. McNair, "SignificantTrends and Developments in the Post-War Period ", in A. Smith (ed.), Competitive Distribution in a Free High Level Economy and Its Implications for the University, University of Pittsburgh Press, 1957. 
Loffire des hard discounters en France

\section{Nombre total de références en magasin}

\section{Nombre de marques nationales}

\section{Surface moyenne d'un magasin}

\section{Nombre de magasins en janvier 2009 (évolution par rapport à 2007)}

\begin{tabular}{|lcccc|}
\hline Aldi & 600 & 3 & $720 \mathrm{~m}^{2}$ & $785(+97)$ \\
\hline Le Mutant & 1300 & 16 & $600 \mathrm{~m}^{2}$ & $220(+24)$ \\
\hline Leader Price (Casino) & 2800 & 23 & $900 \mathrm{~m}^{2}$ & $530(+54)$ \\
\hline Netto (Intermarché) & 1850 & 59 & $710 \mathrm{~m}^{2}$ & $409(+84)$ \\
\hline Lidl & 900 & 165 & $690 \mathrm{~m}^{2}$ & $1450(+185)$ \\
\hline ED (Carrefour) & 2500 & 374 & $700 \mathrm{~m}^{2}$ & $914(+89)$ \\
\hline (Adapté de l'atlas LSA 2009 et de Linéaires, octobre 2007) & & \\
\hline
\end{tabular}

l'acceptation des paiements par carte bancaire. Et l'offre pourrait s'étendre encore : en Allemagne, Lidl a ainsi lancé une carte de fidélité et propose désormais une gamme de téléphonie mobile. Enfin, le hard discount adopte depuis peu des techniques de vente réservées jusqu'alors aux enseignes traditionnelles, avec des promotions ponctuelles, adaptées aux contraintes du format. Elles prennent la forme, par exemple, de lots virtuels de type "moins cher à l'unité si on en achète trois », avec un simple marquage sur le linéaire (sans lot ni modification du packaging) et une réduction qui s'applique automatiquement en caisse. Sans contestation possible, ces évolutions s'inscrivent parfaitement dans le cadre théorique du modèle de la roue de la distribution, et conduisent les observateurs à requalifier certains hard discounteurs comme ED ou Leader Price de « magasins de proximité » qui se positionnent de façon assez proche des supermarchés des années 70. En tant que composante clé du système d'offre du distributeur, la MDD participe activement à ces mouvements stratégiques.

Un embourgeoisement de la MDD. Fin 2009, la part de marché en valeur des
MDD dans les linéaires de la grande distribution alimentaire classique en France atteint $28 \%$ et situe ce pays, selon les données du Panel International d' AC Nielsen, en cinquième position derrière la Suisse (46\%), le Royaume-Uni (40\%), l'Allemagne (30\%) et la Belgique (29\%). Et leur poids est encore plus important si l'on inclut les ventes du hard discount : il est estimé à $40 \%$ en valeur du marché des produits de grande consommation. Les MDD ont connu une forte évolution de leur positionnement au cours du temps. On peut parler de trois, voire quatre générations de MDD, dont la constante évolution est de s'éloigner $\mathrm{du}$ positionnement prix originel pour adopter un positionnement de montée en gamme de plus en plus qualitatif, voire à l'avantgarde du développement durable.

La première génération naît en avril 1976 à l'initiative de Carrefour. Ce sont les fameux «produits libres » portant sur une trentaine de «me too products » vendus à des prix inférieurs de 10 à $30 \%$ à ceux des marques nationales. Le terme de MDD est d'ailleurs impropre car les "produits libres » sont vendus sans marque, dans des 
$\gg$ emballages dépouillés, avec le simple logo et les couleurs de l'enseigne; il serait plus approprié de parler de produits drapeaux ou génériques. La seconde génération date du milieu des années 80 . Appelées cœur de gamme (ou cœur de marché), elles se positionnent comme une offre alternative à la marque de référence du marché, avec un rapport qualité-prix très attractif et un écart de prix de 15 à $20 \%$. Les distributeurs s'engagent en offrant une caution de qualité au consommateur pour gagner sa confiance : ils apposent pour cela leur marque d'enseigne sur les produits. Carrefour est l'initiateur de cette tendance nouvelle en transformant ses «produits libres » en produits Carrefour en 1985. Suivront les produits Champion et

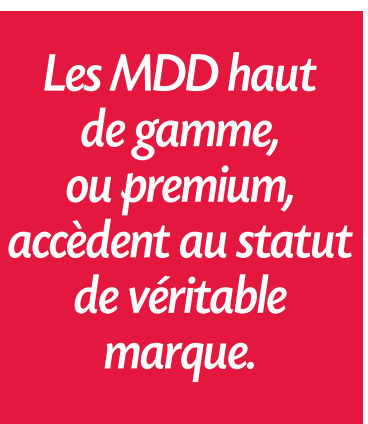
Casino, puis plus tard la marque signifiante Repère de Leclerc, placés sur les rayons à coté des marques nationales, avec un facing équivalent.

La troisième génération de MDD, enfin, s'éloigne du positionnement prix originel. Au milieu des années 90, les distributeurs ont acquis des compétences commerciales, industrielles et logistiques jusque-là maîtrisées par les industriels ${ }^{(2)}$, et ils ont l'ambition de proposer sous MDD des produits innovants qui participent à la stratégie de différenciation de leurs enseignes. Ainsi sont créées des MDD premium, thématiques, avec des axes terroir, bio ou santé. Les exemples sont nombreux comme l'innovation de la lessive liquide en dose lancée par Intermarché début 2001 sous la marque Apta, et rapidement copiée par les fabricants. Des enseignes telles que Casino et Monoprix acquièrent la réputation de «bons lanceurs de produits nouveaux », et l'écart de prix avec les marques nationales se resserre, signe d'un embourgeoisement des MDD. Le choix des noms de MDD traduit l'évolution de leur positionnement : il s'agit de marques propres, exclusives à l'enseigne (et parfois détachées de la marque enseigne), aux noms le plus souvent évocateurs.

Parallèlement, les distributeurs investissent de nouvelles gammes de produits, encore épargnées par la vague des MDD, comme les aliments pour enfants ou les produits cosmétiques. Ces MDD haut de gamme (ou premium) accèdent au statut de véritable marque, avec un territoire d'image et une personnalité propre, un univers imaginaire autonome. Pour preuve, des travaux en marketing sur la marque et le comportement du consommateur commencent à s'intéresser à la valeur perçue des MDD, en distinguant les marques d'enseigne des marques propres des distributeurs ${ }^{(3)}$. Rien à voir donc avec la première génération de MDD pour laquelle il était entendu que les consommateurs ne recherchaient qu'un bénéfice économique et utilitaire, excluant toute motivation d'ordre expérientiel ou symbolique. La MDD premium constitue au contraire un vecteur de communication riche, gratifiant, source de différenciation et de valorisation pour l'enseigne.

On peut voir dans les évolutions récentes l'émergence d'une quatrième génération de MDD, mais ce phénomène ne fait pas l'objet d'un véritable consensus. Certaines MDD deviennent des vecteurs de communication des enseignes, véhiculent les valeurs de l'entreprise, voire se présentent comme une sorte d'outil militant. Par exemple la gamme Agir de Carrefour relaie la communication institutionnelle de l'enseigne vers un engagement désormais fortement médiatisé dans le développement durable. Le niveau de prix est clairement supérieur à celui des

> (2) M. Francis, « New Product Development and Information Technology in Food Supply Chain Management : The Case of Tesco ", in M. Bourlakis et P. Weightman (eds.), Food Supply Chain Management, Blackwell Publishing, 2004. > (3) M. Jara, "Le capital-marque des marques de distributeurs : une approche conceptuelle différenciée ", Revue française du marketing, $\mathrm{n}^{\circ} 221,2009$. 


\section{Les quatre générations de MDD : quelques illustrations}

\begin{tabular}{|c|c|}
\hline Type de MDD & Exemples \\
\hline $\begin{array}{l}\text { Produit premier prix } \\
\text { (nouvelle génération) }\end{array}$ & $\begin{array}{l}\text { Eco+ (Leclerc); Premier prix (Système U); Produits n }{ }^{\circ} 1 \text { (Carrefour); Top Budget } \\
\text { (Intermarché); Pouce (Auchan) }\end{array}$ \\
\hline $\begin{array}{l}\text { MDD cœur de gamme } \\
\text { (Cœur de marché) }\end{array}$ & $\begin{array}{l}\text { Traditionnel } \\
\text { Casino, Champion, Carrefour, Repère (Leclerc) } \\
\text { Nouveaux secteurs } \\
\text { Ysiance [produits de beauté], Tom et Pilou [aliments pour enfants], gamme Ondilège } \\
\text { [produits allégés] (Casino) }\end{array}$ \\
\hline MDD premium & $\begin{array}{l}\text { Terroir/gourmet/plaisir } \\
\text { Reflets de France (Carrefour); Nos régions ont du talent (Leclerc); Club des } \\
\text { sommeliers, Saveurs de toujours, Saveurs gourmandes (Casino); Mmm (Auchan); } \\
\text { Saveurs U (Système U); Gourmet Gault-Millau (Monoprix) } \\
\text { Gammes bio, équitables, exotiques } \\
\text { Monoprix Bio, Bien vivre, Monoprix vert (Monoprix); Casino Bio, Terre et Saveurs } \\
\text { (Casino) } \\
\text { Occasions exceptionnelles } \\
\text { Champion Collection; Carrefour Sélection; Palmarès Casino }\end{array}$ \\
\hline MDD militante & Agir (Carrefour) \\
\hline
\end{tabular}

MDD premium (et les ventes demeurent modestes pour l'instant). Cette nouvelle génération de MDD reste néanmoins très proche de la précédente dans la mesure où elle explore un territoire d'image extrêmement porteur et à forte dimension expérientielle. Le tableau page ci-dessus décline les quatre générations de MDD en donnant différentes illustrations selon les enseignes.

\section{La coexistence de quatre} gammes. Le statut des MDD a évolué dans le temps, avec le lancement de marques plus qualitatives qui ont progressivement assimilé les attributs des marques de fabricant. Quatre gammes de MDD coexistent ainsi sur les linéaires : les premiers prix entrée de gamme, qui ont ressurgi sur les linéaires avec l'essor du hard discount; les MDD cœur de gamme, d'un meilleur rapport qualité/prix que la marque nationale phare; les MDD premium, thématiques et à visée expérientielle; les MDD militantes, qui relaient les valeurs de l'enseigne. Un tel portefeuille de marques, constitué d'un nombre croissant de références, autorise les distributeurs à mener conjointement une stratégie de domination par les coûts, une stratégie de différenciation et une stratégie de niche.

L'analyse des premières campagnes de communication à la télévision, depuis l'ouverture du média aux distributeurs en janvier 2007, souligne cette diversité. Les messages diffusés par les distributeurs mettent largement en scène les MDD, tantôt avec un positionnement prix, tantôt avec un positionnement tourné vers la différenciation, tantôt avec une communication institutionnelle, tantôt avec une communication à finalité commerciale. Signe palpable de la crise que connaissent les pays occidentaux depuis l'automne 2008, le discours publicitaire des enseignes s'infléchit cependant de plus en plus vers la défense du pouvoir d'achat des consommateurs (voir tableau page suivante). La crise semble rebattre les cartes 


\section{Le thème du pouvoir d'achat dans les publicités télévisées en France}

$\begin{array}{ll}\text { Carrefour } & \text { "Il y a des mois où le chèque de fidélité Carrefour tombe à pic. Avec la carte de fidélité } \\ \text { Carrefour, vous recevez chaque mois chez vous le chèque de fidélité Carrefour qui cumule } \\ \text { toutes les économies que vous avez réalisées. Valable chez Carrefour et maintenant } \\ \text { Carrefour Market. " }\end{array}$

Système U Spot 1. « En 2009 chez U, c'est promis, on va encore baisser les prix, mais pas la valeur que I'on accorde à nos clients. U les nouveaux commerçants; avec le U commerce, gardez le pouvoir sur vos achats. "

Spot 2. « Et si en 2009 la qualité des produits premiers prix augmentait, tandis que le prix de grandes marques continuait de baisser, et si votre fidélité devenait encore plus payante, hein? Et si en 2009 vous pouviez contribuer à respecter l'environnement avec des produits qui respectent aussi votre porte-monnaie? Et si la mode devenait vraiment, mais vraiment plus accessible? Et si vous économisiez sur la téléphonie mobile, sans réduire la durée de vos appels? Vous l'avez compris, en 2009, chez U, on va faire baisser beaucoup de choses sauf une : la valeur que l'on accorde à nos clients. Avec le $U$ commerce, gardez le pouvoir sur vos achats. "

Spot 3. "En faisant vos courses ici, vous avez agi là au Liberia, ou encore là en Mongolie, alors merci d'avoir offert vos points carte U à Action contre la faim. Grâce à ces dons, des actions concrètes sont menées sur tous les continents pour sauver des vies et faire tourner le monde un peu plus rond."

Spot 4. " Comment être bien dans son jean, sans faire du mal à son budget? C'est vrai ça, qu'on ait un côté rock bien affirmé, qu'on ait les idées larges, qu'on voie la vie en rose ou même tout en noir... on a tous le droit d'être victime de la mode, mais pas de son prix. C'est pourquoi chez $\mathrm{U}$, il n'y a pas que nos jeans qui sont bien coupés, leur prix aussi. Avec le $\mathrm{U}$ commerce, gardez le pouvoir sur vos achats. »

Intermarché

" Chez Intermarché, on lutte tous contre la vie chère. Chloé, par exemple, elle est Mousquetaire chez Intermarché. En observant les familles, elle s'est vite rendu compte que plus on a d'enfants, plus on dépense d'argent. Alors elle a créé un nouvel avantage à la carte de fidélité, le programme familles nombreuses, $10 \%$ d'économies sur plus de 500 produits de la sélection des Mousquetaires. Et $10 \%$ d'économies sur autant de produits, ça fait des familles $100 \%$ heureuses. Intermarché, tous unis contre la vie chère ".

\section{Analyse thématique}

Six messages sur sept ont pour thème principal la lutte pour le pouvoir d'achat:

- pouvoir d'achat-économies-prix : Carrefour, Leclerc, Système U spots 1, 2 et 4, Intermarché;

- structure de gamme (marques de fabricant-MDD-produits premier prix) : Leclerc, Système Ú spot 2;

Dise en scène des MDD : Leclerc;

Didélité-carte : Carrefour, Système U spot 3, Intermarché;

Daleur client-respect client-étude besoin : Système U spots 1, 2 et 4, Intermarché;

Dactions citoyennes : Système U spot 3;

Donheur : Intermarché.

$\gg$ des stratégies jugées gagnantes, et la MDD est dans le collimateur.

\section{La roue de la marque de distributeur}

La MDD est analysée le plus souvent sous l'angle de la concurrence entre acteurs du canal de distribution, qu'elle soit verticale (entre industriels et distributeurs) ou horizontale (entre enseignes). Il est possible d'appréhender les mutations des MDD sous un autre angle, en tentant de faire un parallèle entre cycle de vie des formats et évolution du positionnement des MDD. En effet, 
arme de concurrence verticale dans la phase de croissance marquée par la multiplication des points de vente, les MDD deviennent une arme de concurrence horizontale dans la phase de maturité et, finalement, une arme de concurrence interformats avec la montée en puissance du hard discount, qui pourrait accélérer le déclin de la distribution traditionnelle de type hypermarché et supermarché, comme le suggère le modèle de la roue de la distribution. Ces différentes étapes ne correspondent-elles pas finalement à une sorte de " roue de la MDD », calqué sur le modèle de la roue de la distribution?

\section{Une arme dans la concurrence verticale.}

De nombreuses recherches s'attachent à expliquer la montée en puissance des MDD sous l'angle du rapport de force, du « bras de fer », entre industriels et distributeurs lors des négociations de référencement ${ }^{(4)}$. Fidèles à leur culture transactionnelle et d'affrontement, les distributeurs auraient utilisé les MDD pour fragiliser la position des industriels les plus puissants et offrir une alternative crédible au consommateur. Face à la rareté des linéaires, introduire une nouvelle gamme de MDD revient en effet à évincer d'autres marques. L'assortiment, souvent constitué d'une ou deux marques de fabricant et de trois MDD (premier prix, cœur de gamme et premium), ne laisse guère de place pour une troisième marque de fabricant, même si, parfois, une marque régionale joue le rôle de «challenger ». D'où des négociations difficiles pour les industriels, conscients de cet état de fait et de la menace corrélative d'un déréférencement partiel ou total.

Sans conteste, la montée en qualité des MDD, avec les MDD cœur de gamme fabriquées par les PME, a été favorisée par la volonté des distributeurs de limiter les hausses des tarifs des industriels. En effet, en cas de hausse des tarifs des marques nationales trop élevée, la réponse des distri- buteurs peut consister à réduire leur place en rayon; il suffit d'installer comme challenger la marque propre d'une PME déjà partenaire pour les MDD et/ou d'étendre le facing des MDD dont le rapport qualité/prix est meilleur. L'ouverture des écrans de télévision aux distributeurs pourrait d'ailleurs exacerber la concurrence verticale : en autorisant la mise en scène des MDD, elle entraîne potentiellement une perte d'efficacité des marques nationales, qui seules jusqu'alors pouvaient s'exprimer dans une stratégie pull, avec une communication destinée à travailler l'image de marque et à développer l'attitude favorable du consommateur à l'égard de la marque.

\section{Une arme dans la concurrence horizon-}

tale. Privés de la possibilité de se différencier par les prix du fait de l'interdiction des pratiques discriminatoires par la loi Galland de juillet 1996, les distributeurs ont cherché de nouvelles voies de différenciation, et ils ont particulièrement bien réussi à travers les MDD cœur de gamme et thématiques. Celles-ci contribuent en effet à façonner l'image et le positionnement des enseignes. Elles leur permettent d'effectuer une adaptation fine de leur offre aux besoins des consommateurs. S'appuyant sur la réactivité de PME partenaires, les enseignes jouent ici sur une capacité à modifier presque instantanément leur gamme de produits pour " coller » aux attentes des clients, adapter leurs produits à des occasions d'achat particulières et, plus généralement, se différencier par un marketing combatif.

La tendance nouvelle se traduit par une hausse non négligeable du prix des MDD, comme le note la revue Que choisir? dans

> (4) M. Filser, V. des Garets et G. Paché, La Distribution : organisation et stratégie, Editions Management \& Société, 2001. 
$\gg$ son numéro de septembre 2008 : « Les MDD représentent désormais plus du tiers des achats des Français. Elles autorisent une économie de $24,3 \%$, toutes enseignes confondues. Mais ces MDD sont aussi les références qui ont le plus augmenté ces huit derniers mois : $+7,7 \%$ en moyenne (et $+8,3 \%$ en hyper). » Les raisons sont à trouver du côté de la flambée des prix des matières premières car moins un produit est sophistiqué, plus le poids des matières premières $y$ pèse fortement. La hausse du prix des MDD résulte aussi (et surtout?) d'un choix de distributeurs soucieux de reconstituer leurs marges.

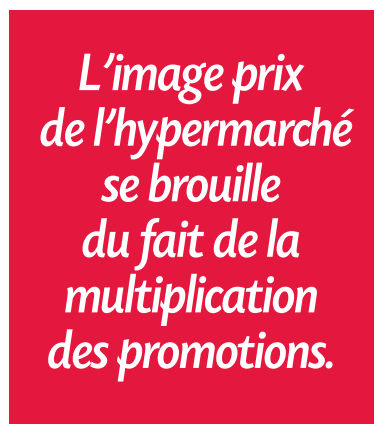

de MDD proposé par les distributeurs (voir figure ci-contre). La montée en qualité des MDD accompagne l'embourgeoisement des formats classiques, les rendant vulnérables à de nouveaux concepts conformément au modèle de la roue de la distribution. L'expansion du hard discount peut ainsi être expliquée par l'arrivée à maturité de l'hypermarché, confirmée par l'analyse économétrique de Gérard Cliquet ${ }^{(6)}$. Elle se caractérise par deux éléments : d'une part, le ralentissement de la croissance quantitative du nombre de points de vente, puis la saturation du parc; d'autre part, la hausse des coûts de structure (et des prix) et l'éloignement par rapport au concept initial. Subissant en outre la volonté des pouvoirs Une dernière explication repose sur la montée en puissance des MDD thématiques (santé et équilibre alimentaire, produits du terroir, protection de l'environnement, etc.), à côté des premiers prix et du cœur de gamme.

Les hausses de prix ont été décidées alors que les MDD deviennent des valeurs de référence à la fois en termes de prix, de qualité, de pertinence et d'innovation. La métaphore du train, suggérée par Jean-Noël Kapferer ${ }^{(5)}$, illustre parfaitement le phénomène : les MDD ne sont plus des wagons tirés par les locomotives que seraient les grandes marques. Au contraire, elles sont porteuses d'innovations importantes et totalement originales portant sur le produit lui-même ou sur son packaging. Il est alors tentant pour les distributeurs de conduire une bataille par les prix sur quelques grandes marques nationales et internationales pour créer du trafic en magasin, façonner l'image de marque de l'enseigne et, ensuite, à l'intérieur de l'espace marchand lui-même, d'orienter le consommateur vers les MDD, largement plus rentables pour eux.

La MDD dans la concurrence entre formats. Un parallèle est possible entre évolution des formats et évolution du portefeuille publics de ralentir puis bloquer la croissance des hypermarchés et supermarchés, la distribution alimentaire va ainsi progressivement passer d'une croissance extensive, c'est-à-dire une logique d'ouverture de nouveaux points de vente, à une croissance intensive ou " qualitative », c'est-à-dire un objectif de hausse du chiffre d'affaires de chaque magasin.

La croissance qualitative se traduit par le lancement de programmes de fidélité mais aussi l'ajout de services, la montée en gamme des produits et des hausses de prix de vente pour couvrir les frais de structure que connaît toute organisation arrivée à maturité. Le développement de MDD plus qualitatives participe du même mouvement. L'image prix de l'hypermarché se dégrade et se brouille, du fait de la multiplication des promotions lancées par les grandes marques. Les autres éléments du concept hypermarché vieillissent : le libre-service, désormais banalisé, n'est plus, non plus qu'un avantage distinctif, le «tout sous le

$>$ (5) J.-N. Kapferer, " Les marques face au hard discount: quelles stratégies? ", Revue française de gestion, $\mathrm{n}^{\circ} 150$, 2004.

> (6) G. Cliquet, " Large Format Retailers : A French Tradition Despite Reactions ", Journal of Retailing and Consumer Services, vol. 7, $n^{\circ}$ 4, 2000. 
même toit » et l'hyperchoix distinctifs. Le consommateur s'épuise dans des surfaces de vente immenses, il préfère une offre plus limitée, mais méticuleusement choisie. Les MDD cœur de gamme trouvent ici une nouvelle légitimité : elles simplifient le choix du consommateur, confronté à un facing impressionnant, puisque l'enseigne a présélectionné des produits en engageant son nom.

Les supermarchés voient leur part de marché progresser dans un premier temps, sous le double effet du resserrement de l'écart de prix avec les hypermarchés et du nouveau critère qualité/temps des consommateurs. Mais ils déclinent ensuite rapidement avec la montée en puissance du hard discount et de son nouveau concept : le libre-service alimentaire avec un personnel réduit, une présentation sommaire, un assortiment limité aux produits de base, des prix bas. Comme nous l'avons souligné, l'apparition de ce nouveau concept s'inscrit parfaitement dans la logique du modèle de la roue de la distribution. Le hard discount émerge et se développe dans un contexte économique défavorable, alors que le consommateur fait face à la montée des prix de produits dont les dépenses sont jugées incompressibles (l'immobilier, l'énergie) ou s'astreint à des arbitrages budgétaires en faveur de nouveaux besoins (la santé, les nouvelles technologies).

Touchant à l'origine des clients à revenu modeste, parfois même des exclus de la société, les hard discounteurs voient rapidement leur taux de pénétration grandir auprès d'autres catégories sociales. Le hard discount connaît ainsi une croissance soutenue, malgré des surfaces de vente limitées du fait de la stricte application de la loi Raffarin de juillet 1996 et de son seuil d'autorisation à 300 mètres carrés. Les stratégies spatiales d'implantation des magasins de 
$\gg$ hard discount sont diverses, mais elles ont pour point commun de chercher à couvrir le plus rapidement possible les territoires visés pour améliorer la productivité des investissements logistiques et des campagnes de communication. Malgré les barrières à l'entrée, le parc de magasins de hard discount poursuit son expansion et s'appuie, fin 2009, sur 4500 points de vente, pour une part de marché des produits de grande consommation d'environ $14 \%$.

\section{Un horizon brouillé sous la pression du hard discount}

Le modèle économique du hard discount crée une rupture dans la montée en gamme des MDD des distributeurs traditionnels. Fondé sur la recherche des coûts les plus bas, ce modèle s'appuie au départ sur un assortiment étroit, qui comprend peu ou pas de marques nationales, mais des MDD ou des produits sans marque. La menace $d u$ hard discount oblige les enseignes classiques à relancer des produits premier prix qui ressemblent fort aux produits drapeaux des années 70 : une qualité correcte, mais inférieure à celle des MDD cœur de gamme. Leclerc est le premier à réagir en 1999 en lançant

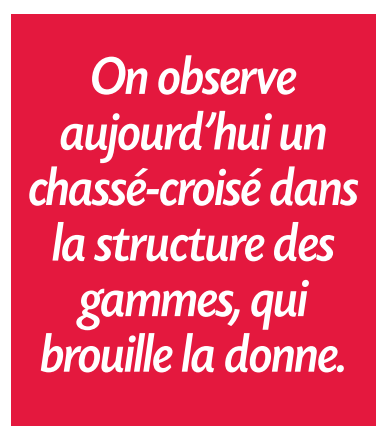

La situation de récession des économies occidentales n'est pas étrangère à ce revirement des distributeurs traditionnels. Une recherche fondée sur une analyse de séries temporelles aux Etats-Unis et en Europe montre que le niveau des ventes de MDD est directement lié à l'état de l'économie ${ }^{(7)}$. Elles augmentent fortement lorsque l'activité se ralentit, puis diminuent lorsqu'il y a reprise économique, même si l'intensité des changements n'est pas symétrique (les consommateurs adoptent rapidement les MDD de type premier prix dès que les difficultés économiques surviennent, mais reviennent lentement aux marques nationales lors de la phase de reprise économique). Les stratégies de développement des MDD s'inscrivent ainsi dans une perspective évolutionniste en fonction de mutations de l'environnement externe, mais aussi en fonction des objectifs poursuivis par les entreprises. Cela ne signifie pas une totale cohérence entre objectifs poursuivis et stratégies mise en œuvre. En effet, on peut aujourd'hui observer un chassé-croisé dans la structure des gammes des formats traditionnels et des hard discounters. Il véhicule un risque non négligeable de brouiller à terme leurs positionnements respectifs aux yeux des consommateurs :

- d'une part, les enseignes tradisa marque premier prix Eco+. Il est suivi par l'ensemble des enseignes des formats traditionnels. Du coup, l'écart de prix entre MDD et marques nationales, qui s'était resserré à $14 \%$ en 2001, se creuse à nouveau. Ajoutons que pour rester en cohérence avec leur communication sur les prix, certains hypermarchés proposent également des produits en vrac sous marque propre, ce qui limite les coûts de distribution et de logistique en permettant de conserver une marge raisonnable malgré une diminution du prix de vente au consommateur. Ceci constitue une illustration supplémentaire (et pertinente) du modèle de la roue de la distribution. tionnelles mettent en avant la structuration de leurs assortiments, comme l'atteste par exemple le spot télévisé de Leclerc début 2009 (voir tableau page 18). Celui-ci oppose les grandes marques de fabricant, les MDD et les produits premier prix, et suggère que Leclerc est moins cher dans les trois segments, conformément au registre habituel de la communication de l'enseigne. Le segment des produits premiers prix est ici distingué, voire opposé à celui des MDD;

> (7) L. Lamey, B. Deleersnyder, M. Dekimpe et J.-B. Steenkamp, « How Business Cycles Contribute to Private-Label Success : Evidence from the United States and Europe ", Journal of Marketing, vol. 71, n 1, 2007. 
- d'autre part, la plupart des hard discounteurs, à l'exception d'Aldi, ont introduit dans leur assortiment de grandes marques, parfois le top of mind, à un prix très inférieur à celui proposé par les hypermarchés et les supermarchés. Celles-ci sont certes peu nombreuses au regard de l'ensemble des références (tout au plus quelques centaines), mais très visibles et largement mises en avant dans la communication d'enseignes comme ED ou Lidl.

La modification de la structure de l'assortiment est-elle une simple tactique pour accélérer le taux de pénétration du hard discount? Ou s'agit-il d'une tendance lourde vers une offre plus qualitative, conformément au modèle de la roue de la distribution? La seconde option semble la plus réaliste, comme en témoignent certaines évolutions récentes. Ainsi, en Allemagne, Lidl commercialise aujourd'hui des MDD équitables sous la marque Fairglobe, et au Royaume-Uni Aldi commercialise des MDD premium sous la marque Specially Selected. Leader Price a ouvert la voie en France avec une gamme de MDD gourmet, et le mouvement pourrait s'accélérer, d'autant que les hard discounteurs français appartiennent tous à des groupes de distribution multi-enseignes qui vendent depuis longtemps les MDD cœur de gamme et premium, et en maîtrisent le processus d'élaboration, le sourcing et la logistique.

Enfin, les nouvelles règles de l'urbanisme commercial issues de la loi de modernisation de l'économie (dite LME) vont manifestement favoriser l'élargissement futur de l'offre des hard discounteurs. Jusqu'alors contraints par le seuil des 300 mètres carrés et les longs délais d'autorisation, les enseignes peuvent, depuis janvier 2009, étendre les surfaces de vente des magasins existants du fait du retour au seuil d'autorisation de 1000 mètres carrés pour toute ouverture ou agrandissement. La surface moyenne des magasins de hard discount pourrait ainsi augmenter rapidement et

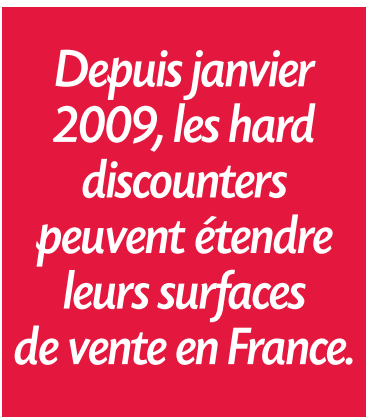
nationales. L'expérience de l'hypermarché hard discount initiée par le groupe Auchan en janvier 2010 est sans doute un premier pas dans cette direction. S'il en allait ainsi, nul doute que la roue continuerait à tourner pour apparenter de plus en plus les hard discounteurs à des supermarchés, voire des hypermarchés, traditionnels, avec un avenir plus que jamais incertain pour les MDD. 